\title{
Erratum to: The sera from adult patients with suggestive signs of autoimmune diseases present antinuclear autoantibodies that cross-react with Leishmania infantum conserved proteins: Crude Leishmania histone and Soluble Leishmania antigens
}

\author{
Sami Lakhal • Meriem Benabid • Ines Ben Sghaier • \\ Jihen Bettaieb · Aïda Bouratbine · Yousr Galai
}

Published online: 21 February 2015

(C) Springer Science+Business Media New York 2015

Erratum to: Immunol Res 2015 61(1-2):154-159

DOI 10.1007/s12026-014-8589-x

An error in the title was inadvertently missed by the author in the original publication, and it has been corrected with this erratum.

Also, the author has updated the authorship, affiliation and acknowledgment section with this erratum.

1. There is a spell error in the title: Soluble Leishmnia antigens should read as Soluble Leishmania antigens.

2. Dr. Jihen Bettiaeb is added as co-author in the fourth position and removed them from the Acknowledgments section.
3. The author has revised the Acknowledgments sections as acknowledged Dr. Manuel Soto. The revised acknowledgments are as follows:

Acknowledgments We would like to thank Dr. Steven G. Reed, Infectious Disease Research Institute (IDRI), Seattle, WA, for providing recombinant $\mathrm{K} 39$ of $L$. infantum. The authors wish also to thank Dr. Randy Howard (IDRI) and Dr. Manuel Soto, Centro de Biología Molecular "Severo Ochoa," Universidad Autónoma de Madrid, Madrid, Spain, for their critical reading of the manuscript.

The online version of the original article can be found under doi:10. 1007/s12026-014-8589-x.

S. Lakhal $(\bowtie) \cdot$ M. Benabid · I. B. Sghaier · A. Bouratbine ·

Y. Galai

Research Laboratory of Medical Parasitology, Biotechnology and Biomolecules, LR 11 IPT06, Pasteur Institute of Tunis, 13, Place Pasteur, B.P. 74, 1002 Tunis, Belvédère, Tunisia

e-mail: sami.lakhal@pasteur.rns.tn

J. Bettaieb

Laboratory of Medical Epidemiology, Pasteur Institute of Tunis,

13, Place Pasteur, B.P. 74, 1002 Tunis, Belvédère, Tunisia

Y. Galai

Laboratory of Clinical Immunology, Pasteur Institute of Tunis,

13, Place Pasteur, B.P. 74, 1002 Tunis, Belvédère, Tunisia 